\title{
BMJ Open Evidence-based interventions to reduce adverse events in hospitals: a systematic review of systematic reviews
}

\author{
Marieke Zegers, ${ }^{1}$ Gijs Hesselink, ${ }^{1}$ Wytske Geense, ${ }^{1}$ Charles Vincent, ${ }^{2}$ \\ Hub Wollersheim ${ }^{1}$
}

To cite: Zegers M,

Hesselink G, Geense W, et al. Evidence-based interventions to reduce adverse events in hospitals: a systematic review of systematic reviews. BMJ Open 2016;6:e012555. doi:10.1136/bmjopen-2016012555

- Prepublication history and additional material is available. To view please visit the journal (http://dx.doi.org/ 10.1136/bmjopen-2016012555).

Received 8 May 2016 Revised 18 July 2016 Accepted 7 September 2016

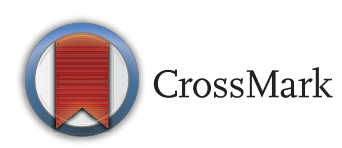

${ }^{1}$ Radboud university medical center, Radboud Institute for Health Sciences, IQ

Healthcare, Nijmegen, The Netherlands

${ }^{2}$ Department of Experimental Psychology, University of Oxford, Oxford, UK

Correspondence to Dr Marieke Zegers; Marieke.zegers@ radboudumc.nl

\section{ABSTRACT}

Objective: To provide an overview of effective interventions aimed at reducing rates of adverse events in hospitals.

Design: Systematic review of systematic reviews. Data sources: PubMed, CINAHL, PsycINFO, the Cochrane Library and EMBASE were searched for systematic reviews published until October 2015.

Study selection: English-language systematic reviews of interventions aimed at reducing adverse events in hospitals, including studies with an experimental design and reporting adverse event rates, were included. Two reviewers independently assessed each study's quality and extracted data on the study population, study design, intervention characteristics and adverse patient outcomes.

Results: Sixty systematic reviews with moderate to high quality were included. Statistically significant pooled effect sizes were found for 14 types of interventions, including: (1) multicomponent interventions to prevent delirium; (2) rapid response teams to reduce cardiopulmonary arrest and mortality rates; (3) pharmacist interventions to reduce adverse drug events; (4) exercises and multicomponent interventions to prevent falls; and (5) care bundle interventions, checklists and reminders to reduce infections. Most $(82 \%)$ of the significant effect sizes were based on 5 or fewer primary studies with an experimental study design.

Conclusions: The evidence for patient-safety interventions implemented in hospitals worldwide is weak. The findings address the need to invest in highquality research standards in order to identify interventions that have a real impact on patient safety. Interventions to prevent delirium, cardiopulmonary arrest and mortality, adverse drug events, infections and falls are most effective and should therefore be prioritised by clinicians.

\section{INTRODUCTION}

Improving patient safety is an ongoing concern for healthcare providers, managers and policymakers. Worldwide, the prevalence of patient harm and death as a result of adverse events is about $10 \%$ among hospitalised patients. Half of these adverse events are considered avoidable. ${ }^{1}$ Despite the

\section{Strengths and limitations of this study}

- This review offers a unique overview of effective patient-safety interventions based on data from systematic reviews, thereby producing a stronger evidence-based oversight of effective interventions compared to the outcomes of a systematic review of primary studies.

- For several patient-safety interventions that are implemented worldwide, there is a lack of highquality studies in which these interventions are evaluated.

- The found estimates of effectiveness of patientsafety interventions might vary across contexts, such as small versus large hospitals, academically affiliated hospitals versus those that are not and the availability of factors that stimulate successful implementation of interventions.

widespread implementation of interventions to reduce patient harm, patient safety is not improving. ${ }^{2-4}$

Substantial effort has been invested into developing and implementing safety improvements. ${ }^{5-7}$ Patient-safety improvement interventions have been defined as: practices, strategies, structures, procedures, behaviour or actions to prevent or mitigate unintended patient harm, resulting from the healthcare process across a range of diseases and procedures. $^{8-11}$ Several reviews have studied the nature and effectiveness of a broad range of these patient-safety interventions. ${ }^{5}$ 12-15 However, the findings of these reviews need to be seen in the light of several limitations. The reviews included studies with weak designs, lacking a systematic approach, or were conducted more than a decade ago. Most importantly, none of the reviews reviewed or prioritised patient-safety interventions based on their effects on adverse event and mortality rates. So far, patientsafety interventions have not been reviewed or prioritised based on effect measures. 
Better insight into the effectiveness of interventions aimed to reduce adverse events and preventable deaths within hospitals is needed to assist managers and healthcare providers with deliberately selecting patient-safety interventions based on available evidence ${ }^{16}$ and to disseminate effective patient-safety improvement interventions into routine practice. ${ }^{3}$ Therefore, the aim of this study was to systematically review systematic reviews of interventions aimed at improving patient safety in hospitals by evaluating interventions, the studies they were tested in and the effect sizes found.

\section{METHODS}

We conducted this systematic review with a prespecified protocol (see online supplementary appendix 1), in accordance with the preferred reporting items for systematic reviews and meta-analyses (PRISMA) and the AMSTAR (A MeaSurement Tool to Assess systematic Reviews) checklist for systematic reviews (see online supplementary appendices 2 and 3$).{ }^{17} 18$

\section{Data sources and searches}

We searched for systematic reviews from inception to 22 July 2013, using the following scientific databases: PubMed (including MEDLINE), CINAHL, PsycINFO, the Cochrane Library and EMBASE. We used the filters for searching papers on patient safety developed by Tanon $e t a l^{19}$ to maximise the sensitivity of our literature search. The search terms used are described in detail in online supplementary appendix 4. We updated the search until 6 October 2015 (see flow chart in figure 1).

Additional hand searches were conducted in high-impact journals and online databases in the field of patient safety, from April 2010 to May 2015, including: Systematic Reviews Journal, Annals of Internal Medicine, BMJ, BMJ Quality and Safety in Healthcare and the International Journal of Quality in Healthcare. Finally, references from the included systematic reviews and bibliographies of published and unpublished reviews related to our study objective were scanned to identify eligible systematic reviews.

\section{Systematic review selection}

Two researchers (MZ and GH) independently assessed the inclusion eligibility of the retrieved systematic reviews according to a standardised format (see online supplementary appendix 1). The initial selection for inclusion was based on the title and abstract of the systematic reviews. A full-text copy of the article was retrieved and reviewed, in case the title and abstract provided insufficient information to determine its relevance. For the final selection, a full-text copy of the systematic reviews was examined to determine whether it fulfilled the inclusion criteria. Disagreement about inclusion was solved by discussion. When no consensus could be achieved, a third reviewer (HW) made the final decision.

Each systematic review had to meet the following criteria (see online supplementary appendix 1):
1. English-language, full-text published and unpublished systematic reviews;

2. including any study matching the Cochrane Effective Practice and Organisation of Care (EPOC) criteria for study designs, including: randomised controlled trials, non-randomised controlled trials, controlled before-after studies and interrupted time series; ${ }^{20}$

3 . focusing on population of hospitalised patients across a range of diseases and procedures;

4. regarding patient-safety interventions (aimed at changing healthcare processes, structures, strategies, behaviour or actions) targeted at reducing adverse events;

5. reporting quantitative effect measures.

Systematic reviews that met any of the flowing criteria were excluded from the review:

1. only obtaining observational studies;

2. only obtaining pharmacological studies;

3. only obtaining psychiatric, obstetric patients or neonates as the study population/sample;

4. only including process errors or consequences of adverse events (eg, readmission and length of stay).

Systematic reviews were included if they included observational studies and studies that met the EPOC criteria. Of these systematic reviews, only the studies that met the EPOC criteria for study designs were studied and were called 'eligible studies'.

\section{Data extraction and quality assessment}

One researcher (WG) extracted the data from the included systematic reviews, using a standardised form (see online supplementary appendix 1). The extracted data were checked by a second researcher (GH). Disagreement was resolved through discussion, and a third person (MZ) was consulted if needed. We limited the data extraction to the prespecified elements, including the intervention components, design and number of included studies, study sample (nature and size) and effect measures. Of all of the studies in a systematic review, only data from studies that met our selection criteria (called 'eligible studies') were extracted and analysed.

Three reviewers (MZ, GH and WG) independently assessed the extent to which the systematic review was conducted to the highest possible standards, using a quality assessment form (see online supplementary appendix 1) that included the 11 AMSTAR quality criteria. ${ }^{18}$ Systematic reviews scored 1 point for each fulfilled criterion, and a total score for each systematic review was calculated. A score of $0-3$ was classified as 'low', 4-7 as 'moderate' and 8-11 as 'high'. ${ }^{21}$

\section{Data synthesis and analysis}

The study characteristics and patient outcomes for all of the systematic reviews that met our inclusion criteria were organised in a tabular form. The systematic reviews included were classified into patient-safety areas. The classification was adapted from previous reviews on patient-safety interventions. ${ }^{1112} 14$ 


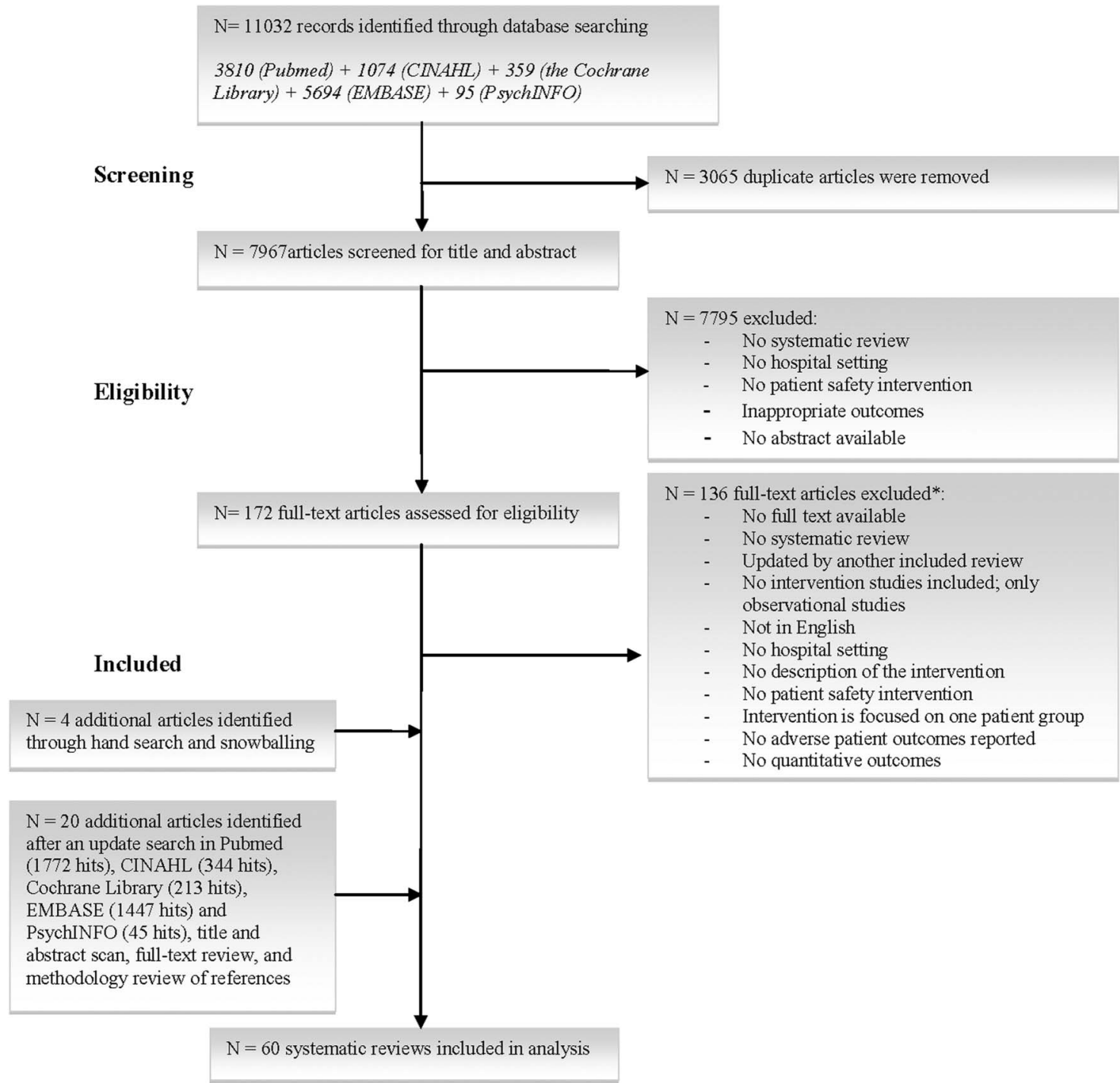

*See Appendix 5 for the exclusion reason per systematic review after full text selection

Figure 1 Summary of evidence search and selection. *See online supplementary appendix 5 for the exclusion reason per systematic review after full-text selection.

The overlap in primary studies between systematic reviews was studied. Systematic reviews of which all included studies were included in a more recent systematic review (100\% overlap) were excluded. We reported the proportion $(\%)$ overlap between included systematic reviews per patient-safety area.

We compiled the pooled effect sizes of meta-analyses reported in the systematic reviews and analysed the intervention components. Subsequently, we ranked the effective interventions based on their effect size.

\section{RESULTS}

\section{Search results}

Our initial search identified 11032 records (figure 1). The title and abstract scan resulted in 172 articles that underwent full-text review. Thirty-six articles met our selection criteria after the full-text review. The exclusion reasons for the 136 articles are given in online supplementary appendix 5 . Four additional articles were identified through hand searching and snowballing, and 20 additional articles were identified through an update of our search action. The final set consisted of 60 articles $^{22-81}$ that underwent data abstraction and analysis.

\section{Methodological quality}

Four $(6.7 \%)$ systematic reviews scored low, $30(50.0 \%)$ scored moderate and $26(43.3 \%)$ scored high on methodological quality. Their AMSTAR scores ranged from 2 to 10 (see online supplementary appendix 6), with a mean score of $6.9(\mathrm{SD} \pm 2.2)$. None of the included systematic reviews fulfilled all of the AMSTAR criteria. Online supplementary appendix 7 shows the proportion of studies satisfying each of the 11 AMSTAR quality criteria. Most $(>80 \%)$ of the included systematic reviews carried out a comprehensive literature search, reported 
the characteristics of the included studies, assessed the scientific quality of the included studies and used the scientific quality of the included studies appropriately in formulating conclusions. One-third of the systematic reviews referred to a study protocol in which the research questions and inclusion criteria were established before the study was conducted, and provided a list of included and excluded studies. None of the systematic reviews reported the conflicts of interest of the included studies (see online supplementary appendix 7). Six systematic reviews $(10.0 \%)$ did not include a statement on the presence or absence of potential conflicting sources of support for carrying out the systematic review. 424546526878

\section{Characteristics of the included systematic reviews}

The characteristics of the included systematic reviews are summarised in online supplementary appendix 8 . More than half $(56.7 \%)$ of the systematic reviews were published between 2013 and 2015. The total number of included studies ranged from $2^{6781}$ to $138 ;^{65}$ the number of eligible studies (ie, met the inclusion criteria) ranged from $1^{678081}$ to $33 .^{29}$ The number of participants in the eligible studies ranged from $938^{75}$ to $225686^{71}$ and was not reported or unknown in $26(43.3 \%)$ reviews.

The included reviews covered 14 patient-safety areas (table 1). Most of the reviews were about preventing adverse drug events $(n=15)$, followed by infection prevention $(\mathrm{n}=8)$, delirium prevention $(\mathrm{n}=7)$ and adverse events after hospital discharge or clinical handover $(\mathrm{n}=7)$.

There was overlap in the included studies between systematic reviews within specific patient-safety areas (see online supplementary appendix 9). The overlap ranges from $25 \%{ }^{45}$ to $86 \%{ }^{47}$ for 'delirium prevention' and from $66 \%{ }^{62}$ to $75 \%{ }^{59}{ }^{60}$ for 'fall prevention'.

\section{Effects of patient-safety interventions}

The results of all included systematic reviews are summarised in online supplementary appendix 10. A meta-analysis was carried out in 30 of the $60(50.0 \%)$ systematic reviews (table 2). The authors addressed the following reasons for not performing a meta-analysis: too few studies identified $(n=5)$; the heterogeneity of the respective study designs $(n=9)$, interventions $(n=8)$, subject groups $(n=5)$ and reported outcomes $(n=5)$; and methodological limitations (eg, lack of available valid data) of the included studies $(n=5)$.

Seventeen meta-analyses showed a statistically significant effect on adverse drug events, ${ }^{36}$ catheterassociated urinary tract infection (CAUTI) rates, ${ }^{40}$ central-line-associated bloodstream infection (CLABSI) rates, ${ }^{39}$ delirium incidence, ${ }^{47} 5051$ fall rates,${ }^{61}$ surgical-site infections, ${ }^{66}$ incidence of cardiopulmonary arrest, ${ }^{69} 71$ complications $^{66} 79$ and mortality rates. ${ }^{33} 415866717576$ Patient-safety interventions with statistically significant effect sizes are discussed below.
Adverse drug event

Of the 15 included systematic reviews about adverse drug events, 2 reported statistically significant results. Davey et $a \hat{l}^{3}$ found that interventions aimed at increasing antibiotic guideline compliance for pneumonia were associated with a significant reduction in mortality: risk ratio (RR) 0.89 (95\% CI 0.82 to $0.97 ; \mathrm{p}=0.01)$. This found effect was based on four studies. Effective intervention components were formal presentations, academic detailing, letters, frequent reminders by pharmaceutical representatives, preprinted outpatient and admission order sheets and reporting of outcome data to providers.

Wang $e t a l^{6}$ found that participation of a pharmacist in physician rounds and timely information exchange and advice of physicians by the pharmacist (ie, on drug interactions, appropriate dosages, dose intervals and routes of administration) was associated with a statistically significant reduced adverse-drug-event rate: OR 0.23 (CI 0.11 to $0.48 ; \mathrm{p}<0.01$ ). The found effect was based on three studies, of which two complied with the Cochrane EPOC inclusion criteria for study designs.

\section{Infection}

Three systematic reviews reported statistically significant effects on the reduction of infection and mortality rates as a result of implementing interventions and care bundles. ${ }^{39-41}$ The meta-analysis performed by Blot $e t a l^{39}$ showed a reduction in the CLABSI rate (OR 0.39 (CI 0.33 to $0.46 ; \mathrm{p}<0.01)$ ) and reduction in the CLABSI rate at 3 months post intervention (OR 0.30 (CI 0.10 to $0.88 ; \mathrm{p}=0.028)$ ) as a result of care bundles and checklists. ${ }^{39}$ These found effects were based on 41 and 6 studies, respectively, of which 5 and 4 studies met our inclusion criteria, respectively.

Meddings $e t a t^{40}$ reported that the use of a reminder and/or stop order to prompt removal of unnecessary urinary catheters led to a $53 \%$ reduction of CAUTI episodes per 1000 catheter days: rate ratio (RaR) 0.47 (CI 0.30 to $0.64 ; \mathrm{p}<0.01$ ). This meta-analysis was based on 11 studies, of which only 1 study complied with the inclusion criteria for study designs.

The implementation of a programme to improve compliance to sepsis care bundles led to a statistically significant decreased mortality rate: OR 0.66 (CI 0.61 to 0.72 ; $\mathrm{p}<0.01)$. This rate is based on 48 studies, of which 3 fulfilled the criteria for study designs. ${ }^{41}$

\section{Delirium}

Three systematic reviews reported a statistically significant reduction in delirium incidence. ${ }^{475051}$ There was a $16 \%$ overlap ( 3 of the 19 studies) between these systematic reviews (see online supplementary appendix 9 ).

Hempenius et al pooled the effects of five studies and found a statistically significant effect of multicomponent interventions to prevent delirium: OR 0.58 (CI 0.38 to 0.92$)$. Components were education, systematic cognitive screening, geriatric consultative services, supportive psychotherapy and a scheduled pain protocol. 
Table 1 Identified systematic reviews $(n=60)$ classified by patient-safety area $(n=14)$

\begin{tabular}{|c|c|c|c|}
\hline \multicolumn{2}{|c|}{ Patient-safety area } & \multirow[t]{2}{*}{$\begin{array}{l}\text { Number of } \\
\text { systematic reviews } \\
\text { (references) }\end{array}$} & \multirow[t]{2}{*}{$\begin{array}{l}\text { Intervention components relevant to patient safety } \\
\text { (effective components are in bold) }\end{array}$} \\
\hline Adverse & Subarea & & \\
\hline \multirow[t]{4}{*}{ drug event } & CPOE system & $2^{22} 23$ & CPOE system \\
\hline & Medication review & $4^{24-27}$ & Medication reconciliation \\
\hline & $\begin{array}{l}\text { Computer-assisted decision } \\
\text { support/alerts }\end{array}$ & $3^{28-30}$ & $\begin{array}{l}\text { Computerised advice or decision support; } \\
\text { computerised drug-laboratory alerts for clinicians on } \\
\text { prescribing or monitoring decisions }\end{array}$ \\
\hline & $\begin{array}{l}\text { Multicomponent } \\
\text { interventions }\end{array}$ & $6^{31-36}$ & $\begin{array}{l}\text { Multicomponent interventions, including } \\
\text { pharmacist involvement and support of care } \\
\text { teams or physicians; guideline implementation, } \\
\text { including academic detailing, reminders and } \\
\text { feedback of data; multicomponent intervention, } \\
\text { including CPOE system, changes in work schedules, } \\
\text { education, support systems for clinical } \\
\text { decision-making }\end{array}$ \\
\hline \multirow[t]{4}{*}{ Infection* } & $\begin{array}{l}\text { Device-related infections } \\
\text { (CAUTI; CLABSI; VAP) }\end{array}$ & $4^{37-40}$ & $\begin{array}{l}\text { Care bundles and checklists; empowerment to stop } \\
\text { procedure; surveillance; infrastructure and } \\
\text { organisational changes; training on appropriate } \\
\text { catheter placement; catheter restriction and } \\
\text { removal protocols; reminder or stop order to } \\
\text { decrease catheter placement; use of specific } \\
\text { technologies }\end{array}$ \\
\hline & Sepsis & $1^{41}$ & $\begin{array}{l}\text { Multicomponent programme aimed at improving } \\
\text { compliance to sepsis care bundles, including } \\
\text { education and decision support tools }\end{array}$ \\
\hline & Hand-hygiene compliance & $2^{4243}$ & $\begin{array}{l}\text { Education; audit and feedback; health promotion; } \\
\text { variations in the availability and type of products used } \\
\text { for hand hygiene }\end{array}$ \\
\hline & $\begin{array}{l}\text { Overall hospital-acquired } \\
\text { infection }\end{array}$ & $1^{44}$ & Education; protocols to remove catheters \\
\hline Delirium & & $7^{45-51}$ & $\begin{array}{l}\text { Psychiatric assessment; special care; daily visits by a } \\
\text { liaison nurse; interdisciplinary team; supportive } \\
\text { psychotherapy; multicomponent intervention, } \\
\text { including cognitive screening, proactive geriatric } \\
\text { consultation and psychotherapy; multicomponent } \\
\text { intervention, including early mobility, cognition } \\
\text { and orientation, sleep-wake cycle preservation; } \\
\text { multicomponent intervention, including } \\
\text { physiotherapy, family involvement and staff/ } \\
\text { family-member education }\end{array}$ \\
\hline \multicolumn{2}{|c|}{$\begin{array}{l}\text { Adverse event after hospital discharge or } \\
\text { clinical handover }\end{array}$} & $7^{52-58}$ & $\begin{array}{l}\text { Postacute intermediate care units; geriatric } \\
\text { assessment; liaison nurse; predischarge assessment } \\
\text { of risks; patient engagement; individualised patient } \\
\text { record; multidisciplinary discharge planning team; } \\
\text { clinical follow-up; nurse-led early-discharge } \\
\text { planning programmes }\end{array}$ \\
\hline \multicolumn{2}{|l|}{ Fall } & $4^{59-62}$ & $\begin{array}{l}\text { Addressing risk factors by a multidisciplinary } \\
\text { team; care planning; environmental changes; } \\
\text { movement alarms; physiotherapy; management of } \\
\text { urinary incontinence; multicomponent interventions, } \\
\text { including risk alert card, exercise, education, hip } \\
\text { protectors and geriatric assessment }\end{array}$ \\
\hline \multicolumn{2}{|c|}{ Adverse event in surgery } & $5^{63-67}$ & $\begin{array}{l}\text { Screening and decolonisation of surgical-site } \\
\text { infections; subspecialisation; benchmarking; } \\
\text { technology or training; surgical safety checklist }\end{array}$ \\
\hline \multicolumn{2}{|c|}{ Cardiopulmonary arrest } & $4^{68-71}$ & $\begin{array}{l}\text { Critical-care outreach service; rapid response } \\
\text { teams }\end{array}$ \\
\hline
\end{tabular}


Table 1 Continued

\begin{tabular}{|c|c|c|}
\hline Patient-safety area & $\begin{array}{l}\text { Number of } \\
\text { systematic reviews } \\
\text { (references) }\end{array}$ & $\begin{array}{l}\text { Intervention components relevant to patient safety } \\
\text { (effective components are in bold) }\end{array}$ \\
\hline Venous thromboembolism & $2^{7273}$ & $\begin{array}{l}\text { Alerts and education; real-time audit and feedback; } \\
\text { multicomponent interventions to improve appropriate } \\
\text { administration of thromboprophylaxis }\end{array}$ \\
\hline Staffing & $3^{74-76}$ & $\begin{array}{l}\text { Increasing proportion of support staff; addition of } \\
\text { specialist nursing post to staffing; reducing shift } \\
\text { length; protected sleep time; night float; education } \\
\text { among residents; interdisciplinary team } \\
\text { interventions }\end{array}$ \\
\hline Pressure ulcer & $1^{77}$ & $\begin{array}{l}\text { Standardisation of interventions; multidisciplinary } \\
\text { teams and leadership; designated skin champions; } \\
\text { education; audit and feedback }\end{array}$ \\
\hline Mechanical complication and underfeeding & $1^{78}$ & $\begin{array}{l}\text { Total parenteral nutrition team: nutrition support for } \\
\text { patients who are unable to obtain adequate nutrition } \\
\text { either via the oral or enteral route }\end{array}$ \\
\hline Clinical pathway & $1^{79}$ & $\begin{array}{l}\text { Clinical pathways: multidisciplinary care plans with } \\
\text { essential steps in care, supporting the translation of } \\
\text { clinical guidelines into local protocols and application } \\
\text { in practice }\end{array}$ \\
\hline Safety culture & $1^{80}$ & $\begin{array}{l}\text { Error-prevention training; restructured patient-safety } \\
\text { governance; lessons-learnt programme; cause } \\
\text { analysis programme; executive rounds }\end{array}$ \\
\hline External inspection & $1^{81}$ & $\begin{array}{l}\text { External inspections of compliance with standards } \\
\text { (eg, accreditation) }\end{array}$ \\
\hline
\end{tabular}

Hshieh et $a \tilde{p}^{0}$ reviewed studies evaluating nonpharmacological interventions, including the following components: early mobility, cognition and orientation, sleep-wake cycle preservation, hydration, hearing and vision. They found a statistically significant reduction in delirium incidence: OR 0.47 (CI 0.38 to 0.58 ); $\mathrm{p}<0.01$. This rate was based on 11 studies, of which 7 complied with the inclusion criteria for study designs.

Martinez $e t a l^{1}$ found a statistically significant reduction in delirium incidence: RR 0.73 (CI 0.63 to 0.85 ); $\mathrm{p}<0.01$. This rate was based on seven studies, using different multicomponent interventions, but a number of specific components were shared: physiotherapy, daily reorientation, family involvement in care, stimulation programmes with avoidance of sensorial deprivation and staff/family-member education.

\section{Adverse event after hospital discharge or clinical handover}

Six systematic reviews pooled the effect of interventions to improve clinical handover or hospital discharge. One systematic review reported a statistically significant effect size: nurse-led early-discharge planning programmes were associated with a lower mortality rate: RR 0.70 (CI 0.52 to $0.95 ; \mathrm{p}=0.02) .{ }^{58}$ This found effect was based on five studies. Effective intervention components were an individual discharge plan to address identified transitional care needs, comprehensive discharge plan and home-based follow-up visits or telephone calls by providers to patients after their hospital discharge.

Fall

One systematic review ${ }^{61}$ reported the effectiveness of fall-prevention interventions. Additional physiotherapy reduced the risk of falling: RR 0.36 (CI 0.14 to 0.93 ). Multicomponent interventions reduced the fall rate: RaR 0.69 (CI 0.49 to 0.96 ). These rates were based on two and four studies, respectively. Effective components of the multifactorial interventions were fall-risk alert card and information brochure, exercise programme, education programme, hip protectors, comprehensive geriatric assessment and treatment of fall-risk factors by a multidisciplinary team.

\section{Surgical adverse event}

The implementation of a surgical checklist was associated with a reduction of complications, deaths and surgical-site infections: RR 0.59 (CI 0.47 to 0.74 ), 0.77 (CI 0.60 to 0.98 ) and 0.57 (CI 0.41 to 0.79 ), respectively. These pooled rates were based on five studies. ${ }^{66}$ The authors reported that the results were statistically significant but cannot be regarded as definitive in the absence of high-quality studies. ${ }^{66}$

\section{Cardiopulmonary arrest}

Two systematic reviews found an association between the implementation of a rapid response team and improved 
Table 2 Effect sizes of patient-safety interventions: results from meta-analyses $(n=30)$ reported in the 60 included systematic reviews

\begin{tabular}{|c|c|c|c|c|c|c|}
\hline Patient-safety area & $\begin{array}{l}\text { Reference } \\
\text { meta-analysis }\end{array}$ & Intervention & Patient outcome & $\begin{array}{l}\text { significant effect sizes } \\
\text { are bold }\end{array}$ & $\begin{array}{l}p \\
\text { Value }\end{array}$ & $\begin{array}{l}\text { meta-analysis }(n) \\
\text { (eligible studies* }(n))\end{array}$ \\
\hline Adverse drug event & Holland et $a^{P^{4}}$ & Pharmacist-led medication review & Mortality & RR 0.96 (0.82 to 1.13$)$ & 0.62 & 22 \\
\hline \multirow[t]{2}{*}{ Medication review } & $\begin{array}{l}\text { Christensen and } \\
\text { Lundh }^{26}\end{array}$ & Medication review & Mortality & RR 0.98 (0.78 to 1.23$)$ & 0.86 & 4 \\
\hline & Hohl et $a^{R^{7}}$ & Medication review & Mortality & OR 1.09 (0.69 to 1.72$)$ & 0.71 & 3 \\
\hline Adverse drug event & Durieux et $a^{88}$ & Computerised advice on drug dosage & Mortality & RR 0.81 (0.37 to 1.81$)$ & 0.61 & 6 \\
\hline \multirow{2}{*}{$\begin{array}{l}\text { Computerised advice on } \\
\text { drug dosage }\end{array}$} & Gillaizeau et $a^{29}$ & Computerised advice on drug dosage & Mortality & RR 1.08 (0.80 to 1.45$)$ & 0.61 & 10 \\
\hline & Bayoumi et a ${ }^{\beta 0}$ & Computerised drug-laboratory alerts & $\begin{array}{l}\text { Adverse events } \\
\text { (bleeding and } \\
\text { thrombosis) }\end{array}$ & OR 0.88 (0.78 to 1.00$)$ & 0.05 & 4 \\
\hline Adverse drug event & Davey et $a^{\beta 3}$ & Intervention for antimicrobial therapy & Mortality & RR 0.92 (0.69 to 1.22$)$ & 0.56 & 3 \\
\hline Multicomponent & & Antibiotic guideline for pneumonia & Mortality & RR 0.89 (0.82 to 0.97$)$ & 0.01 & 4 \\
\hline \multirow[t]{2}{*}{ interventions } & & Decrease excessive prescribing & Mortality & RR 0.92 (0.81 to 1.06$)$ & 0.25 & 11 \\
\hline & Wang et $a^{\beta 6}$ & Pharmacist interventions & $\begin{array}{l}\text { Preventable adverse } \\
\text { drug events }\end{array}$ & OR 0.23 (0.11 to 0.48$)$ & $<0.01$ & $3(2)$ \\
\hline \multirow[t]{5}{*}{ Infections } & Blot et $a{ }^{\beta 9}$ & Care bundle/checklist interventions & CLABSI & OR 0.39 (0.33 to 0.46$)$ & $<0.01$ & $41(5)$ \\
\hline & & & CLABSI rate at 3 months & OR 0.30 (0.10 to 0.88$)$ & 0.03 & $6(4)$ \\
\hline & Meddings et $a{ }^{40}$ & Catheter reminder and stop order & $\begin{array}{l}\text { CAUTI episodes per } \\
1000 \text { catheter days }\end{array}$ & RR 0.47 (0.30 to 0.64$)$ & $<0.01$ & $11(1)$ \\
\hline & & & CAUTI & RR 0.72 (0.52 to 0.99$)$ & 0.05 & $8(2)$ \\
\hline & Damiani et $a{ }^{41}$ & Sepsis bundle & Mortality & OR 0.66 (0.61 to 0.72$)$ & $<0.01$ & $48(3)$ \\
\hline \multirow[t]{4}{*}{ Delirium } & $\begin{array}{l}\text { Hempenius } \\
\text { et } a f^{47}\end{array}$ & $\begin{array}{l}\text { Multicomponent interventions, including } \\
\text { cognitive screening, proactive geriatric } \\
\text { consultation and psychotherapy }\end{array}$ & Incidence of delirium & OR 0.58 ( 0.38 to 0.92$)$ & NR & 5 \\
\hline & & One-component interventions & Incidence of delirium & OR 1.05 (0.09 to 11.57$)$ & NR & 2 \\
\hline & Hshieh et $a^{50}$ & $\begin{array}{l}\text { Multicomponent intervention, including } \\
\text { early mobility, cognition and orientation }\end{array}$ & Incidence of delirium & OR 0.47 (0.38 to 0.58$)$ & $<0.01$ & $11(7)$ \\
\hline & Martinez et $a^{51}$ & $\begin{array}{l}\text { Multicomponent intervention, including } \\
\text { physiotherapy, daily reorientation, family } \\
\text { involvement and staff/family-member } \\
\text { education }\end{array}$ & Incidence of delirium & RR 0.73 (0.63 to 0.85$)$ & $<0.01$ & 7 \\
\hline \multirow{8}{*}{$\begin{array}{l}\text { Adverse event after } \\
\text { hospital discharge or } \\
\text { clinical handover }\end{array}$} & Griffiths et $a^{{ }^{2}}$ & Nursing-led inpatients units & Mortality & OR 1.10 (0.56 to 2.16$)$ & 0.64 & 7 \\
\hline & & & $\begin{array}{l}\text { Mortality } 3 \text { or } 6 \text { months } \\
\text { post admission }\end{array}$ & OR 0.96 (0.63 to 1.47$)$ & 0.62 & 6 \\
\hline & Conroy et $a^{53}$ & Comprehensive geriatric assessment & Mortality & RR 0.92 (0.55 to 1.52$)$ & 0.77 & 5 \\
\hline & Niven et $a F^{4}$ & Critical-care transition programmes & Mortality & RR 0.84 (0.66 to 1.05$)$ & 0.1 & $3(2)$ \\
\hline & Shepperd et $a^{56}$ & Discharge planning from hospital to & Mortality at $6-9$ months & RR 1.00 (0.79 to 1.26$)$ & 0.69 & 6 \\
\hline & & home & Falls & RR 0.87 (0.50 to 1.49$)$ & 0.61 & 1 \\
\hline & Lowthian et $a^{{ }^{7}}$ & Optimised ED discharge & $\begin{array}{l}\text { Mortality up to } \\
18 \text { months postdischarge }\end{array}$ & OR 1.01 (0.70 to 1.47$)$ & 0.94 & 2 \\
\hline & Zhu et $a^{58}$ & Nurse-led early-discharge planning & Mortality & RR 0.70 (0.52 to 0.95$)$ & 0.02 & 5 \\
\hline
\end{tabular}


Table 2 Continued

\begin{tabular}{|c|c|c|c|c|c|c|}
\hline Patient-safety area & $\begin{array}{l}\text { Reference } \\
\text { meta-analysis }\end{array}$ & Intervention & Patient outcome & $\begin{array}{l}\text { Effect size }(95 \% \mathrm{Cl}) \\
\text { significant effect sizes } \\
\text { are bold }\end{array}$ & $\begin{array}{l}p \\
\text { Value }\end{array}$ & $\begin{array}{l}\text { Studies in } \\
\text { meta-analysis (n) } \\
\text { (eligible studies* }(n))\end{array}$ \\
\hline \multirow[t]{8}{*}{ Fall } & \multirow[t]{3}{*}{ Oliver et $a^{59}$} & \multirow[t]{3}{*}{ Multicomponent intervention } & Falls & RaR 0.82 (0.68 to 1.00$)$ & NR & 12 \\
\hline & & & Fallers & RR 0.95 (0.71 to 1.27$)$ & NR & 12 \\
\hline & & & Fractures & RaR 0.59 (0.22 to 1.58$)$ & NR & 12 \\
\hline & \multirow{2}{*}{$\begin{array}{l}\text { Coussement } \\
\text { et afo }\end{array}$} & \multirow[t]{2}{*}{ Multicomponent intervention } & Falls & RR 0.82 (0.65 to 1.03$)$ & NR & 4 \\
\hline & & & Number of fallers & RR 0.87 (0.70 to 1.08$)$ & NR & 4 \\
\hline & \multirow[t]{3}{*}{ Cameron et $a^{\lceil 1}$} & \multirow[t]{2}{*}{ Multicomponent interventions } & Rate of falls & RaR 0.69 (0.49 to 0.96$)$ & 0.03 & 4 \\
\hline & & & Risk of falling & RR 0.71 (0.46 to 1.09$)$ & 0.12 & 3 \\
\hline & & Exercises & Risk of falling & RR 0.36 (0.14 to 0.93$)$ & 0.04 & 2 \\
\hline \multirow[t]{3}{*}{ Adverse event in surgery } & \multirow[t]{3}{*}{ Bergs et $a^{\beta 6}$} & \multirow{3}{*}{ WHO surgical safety checklist } & Any complication & RR 0.59 (0.47 to 0.74$)$ & $<0.01$ & 5 \\
\hline & & & Mortality & RR 0.77 (0.60 to 0.98$)$ & 0.04 & $4(3)$ \\
\hline & & & Surgical-site infections & RR 0.57 (0.41 to 0.79$)$ & $<0.01$ & 5 \\
\hline \multirow[t]{4}{*}{ Cardiopulmonary arrest } & \multirow[t]{2}{*}{ Chan et $a^{69}$} & \multirow[t]{2}{*}{ Rapid response team } & Mortality & RR 0.92 (0.82 to 1.04$)$ & NR & 16 \\
\hline & & & Cardiopulmonary arrest & RR 0.65 (0.55 to 0.77$)$ & NR & 16 \\
\hline & \multirow[t]{2}{*}{ Maharaj et $a l^{71}$} & \multirow[t]{2}{*}{ Rapid response team } & Mortality & RR 0.91 (0.85 to 0.97$)$ & $<0.01$ & 4 \\
\hline & & & Cardiopulmonary arrest & RR 0.74 (0.56 to 0.98$)$ & 0.04 & 2 \\
\hline \multirow[t]{3}{*}{ Venous thromboembolism } & \multirow[t]{3}{*}{ Kahn et $a l^{72}$} & Alerts & $\begin{array}{l}\text { All venous } \\
\text { thromboembolism }\end{array}$ & RR 0.85 (0.49 to 1.46$)$ & NR & 3 \\
\hline & & \multirow[t]{2}{*}{ Multicomponent interventions } & $\begin{array}{l}\text { All venous } \\
\text { thromboembolism }\end{array}$ & RR 1.01 (0.51 to 1.98$)$ & NR & 5 \\
\hline & & & $\begin{array}{l}\text { Symptomatic deep vein } \\
\text { thromboembolism }\end{array}$ & RR 0.59 (0.18 to 1.98$)$ & NR & 3 \\
\hline \multirow[t]{7}{*}{ Staffing } & \multirow[t]{5}{*}{ Butler et $a l^{75}$} & \multirow{2}{*}{$\begin{array}{l}\text { Addition of specialist nursing post to } \\
\text { staffing }\end{array}$} & In-hospital mortality & RR 0.96 (0.59 to 1.56$)$ & 0.86 & 1 \\
\hline & & & $\begin{array}{l}\text { Postdischarge adverse } \\
\text { events }\end{array}$ & RR 1.03 (0.70 to 1.53$)$ & 0.87 & 1 \\
\hline & & \multirow{3}{*}{$\begin{array}{l}\text { Increasing the proportion of support } \\
\text { staff }\end{array}$} & Mortality in trauma unit & RR 0.41 (0.16 to 1.01$)$ ) & 0.05 & 1 \\
\hline & & & Mortality in hospital & RR 0.56 (0.29 to 1.09$)$ & 0.09 & 1 \\
\hline & & & Mortality at 4 months & RR 0.57 (0.34 to 0.95$)$ & 0.03 & 1 \\
\hline & \multirow[t]{2}{*}{ Pannick et $a l^{76}$} & Interdisciplinary teams & Mortality & wRR 0.92 (0.82 to 1.05$)$ & NR & 7 \\
\hline & & Team practice interventions & Mortality & wRR 0.67 (0.45 to 0.99$)$ & NR & 2 \\
\hline \multirow[t]{3}{*}{ Clinical pathway } & \multirow[t]{3}{*}{ Rotter et $a l^{79}$} & \multirow[t]{3}{*}{ Clinical pathway } & Mortality & OR 0.84 (0.64 to 1.11$)$ & 0.23 & 3 \\
\hline & & & $\begin{array}{l}\text { Complications up to } \\
3 \text { months }\end{array}$ & OR 0.31 (0.13 to 0.72$)$ & 0.07 & 1 \\
\hline & & & In-hospital complications & OR 0.58 (0.36 to 0.94$)$ & 0.03 & 5 \\
\hline
\end{tabular}

*Study design in accordance with methodological criteria of the Cochrane EPOC review group and quantitative data on adverse event rates were reported.

CAUTI, catheter-associated urinary tract infection; CLABSI, central-line-associated bloodstream infection; EPOC, Effective Practice and Organisation of Care; NR, not reported; RaR, rate ratio; $\mathrm{RR}$, risk/relative ratio; wRR, weighted risk ratio. 
patient outcomes. There is an $11 \%$ overlap (2 of the 19 studies) between these systematic reviews (see online supplementary appendix 9). Chan et a $t^{69}$ performed a meta-analysis on 16 studies and found a statistically significant reduction of cardiopulmonary arrests outside the intensive care unit, following the implementation of the rapid response team: RR 0.65 (CI 0.55 to 0.77 ). The authors of the systematic review raised questions about the effectiveness of rapid response team implementation given the lack of an effect of rapid response teams on mortality.

The systematic review of Maharaj $e t a l^{71}$ found a statistically significant reduction in cardiopulmonary arrests based on two studies: RR 0.74 (CI 0.56 to 0.98 ; $\mathrm{p}=0.04$ ) and a statistically significant reduction of deaths based on four studies: RR 0.91 (CI 0.85 to $0.97 ; \mathrm{p}<0.01$ ).

\section{Staffing}

Butler et $a l^{75}$ found 6202 studies that were potentially relevant to studying the effect of hospital-nurse staffing models on mortality and adverse events. However, one study reported a statistically significant effect: increasing the proportion of support staff (ie, dietetic assistants) reduced mortality at 4 months: RR 0.57 (CI 0.34 to 0.95 ; $\mathrm{p}=0.03$ ). The authors stated that they were unable to draw conclusions because of the small number of eligible studies.

Pannick $e t a l^{76}$ found that interdisciplinary team interventions reduced mortality rates: RR 0.67 (CI 0.45 to 0.99). The finding was based on two studies. Effective intervention components were interdisciplinary rounds, including physician, nurse, pharmacist, nutritionist and social worker; expanded senior clinical nurse roles; incorporating structured detailed assessments of premorbid functional and social patient data and investment in allied health professionals as consistent staff members.

\section{Clinical pathway}

Rotter $e t a l^{79}$ found an association between the use of clinical pathways and a reduction of in-hospital complications, based on five studies: OR 0.58 (CI 0.36 to 0.94). Examples of reported complications were postoperative confusion, infection, uncontrolled bleeding and deep vein thrombosis, ventilator-associated pneumonia, joint dislocation and decreased postdischarge mobility up to 3 months postsurgery. The OR for complications up to 3 months, based on one study, was 0.31 (CI 0.13 to 0.72 ).

\section{Summary of effective patient-safety interventions}

Patient-safety interventions that result in a significant reduction in adverse event or mortality rates are presented in table 3 .

Exercises to reduce the risk of falling, surgical safety checklist to reduce the rate of surgical-site infection, rapid response team to prevent cardiopulmonary arrest and multicomponent interventions to prevent delirium have significantly better results compared to changes in staffing and interventions to improve hospital discharge to prevent mortality. Pharmacist interventions and care bundle interventions and checklists were significantly associated with, respectively, reduced rates of adverse drug events and infection rates. These effect measures are, however, partly based on experimental studies (table 3).

Fourteen of the 17 significant effect sizes (82.4\%) were based on five or fewer studies that comply with the inclusion criteria for study design. The effect measures were based on sample sizes varying from 83 to 1143495 patients, for exercises to reduce the risk of falling and rapid response team to reduce the rate of cardiopulmonary arrest, respectively (table 3). The AMSTAR scores of the systematic reviews of the 17 effective patient-safety interventions ranged from 4 to 10 , with a mean score of $7.5(\mathrm{SD} \pm 1.9)$.

Three systematic reviews evaluated multicomponent interventions to prevent delirium (all with different compositions of the multicomponent intervention and different effect measures); two systematic reviews evaluated the effects of rapid response teams, resulting in 14 unique patient interventions (box 1).

\section{DISCUSSION}

We systematically reviewed the literature for effective interventions aimed at reducing adverse event rates and preventable deaths in hospitals. The results showed that there were 14 effective patient-safety interventions (box 1), including: multicomponent interventions to prevent delirium; rapid response teams to reduce cardiopulmonary arrest and mortality rates; exercises and multicomponent interventions to reduce the risk of falling and surgical safety checklist to reduce the rate of surgical-site infection. Other effective interventions were pharmacist interventions to reduce adverse drug events, care bundles and checklists to reduce infection and mortality rates, changes in staffing and interventions to improve hospital discharge to reduce mortality rates. The evidence base that supports the interventions is moderate because $82 \%$ of the found effect measures were based on five or fewer primary studies that fulfilled the Cochrane EPOC criteria for study designs. ${ }^{20}$

This review offers a unique overview of effective patient-safety interventions based on data that are synthesised from systematic reviews, thereby producing a stronger evidence-based oversight of effective interventions compared to the outcomes of a systematic review of primary studies. ${ }^{16}$ The overlap of primary studies in existing reviews is analysed to minimise the potential effects of 'double-counting' primary studies in multiple reviews. ${ }^{82}$ Moreover, most of the systematic reviews included in our review were of high methodological quality (mean AMSTAR score of 6.9 for all included reviews and 7.5 for the reviews with positively pooled outcome effects), thereby increasing the credibility and validity of our findings. ${ }^{18}$

Despite the growing number of experimental studies evaluating the effectiveness of patient-safety interventions, our findings show that the evidence base for 
Table 3 Effective patient-safety interventions $\left(n=14^{*}\right)$

\begin{tabular}{|c|c|c|c|c|c|}
\hline $\begin{array}{l}\text { Intervention effect estimates based on } \\
\text { meta-analysis with only eligible studies } \dagger\end{array}$ & Patient outcome & Effect size $(95 \% \mathrm{Cl})$ & Sample size (n patients) & $\begin{array}{l}\text { Study size } \\
\text { (n studies) }\end{array}$ & $\begin{array}{l}\text { Designs of studies } \\
\text { (n) }\end{array}$ \\
\hline Surgical safety checklist ${ }^{66}$ & $\begin{array}{l}\text { Surgical-site } \\
\text { infections }\end{array}$ & RR 0.57 (0.41 to 0.79$)$ & 15198 & 5 & ITS (5) \\
\hline Rapid response team ${ }^{69}$ & $\begin{array}{l}\text { Cardiopulmonary } \\
\text { arrest }\end{array}$ & RR 0.65 (0.55 to 0.77 ) & 1143495 & 16 & $\begin{array}{l}\text { Non-RCT (2); CBA } \\
\text { (12); ITS (2) }\end{array}$ \\
\hline $\begin{array}{l}\text { Nurse-led early-discharge planning } \\
\text { programmes }^{58}\end{array}$ & Mortality & RR 0.70 (0.52 to 0.95 ) & 2503 & 5 & RCT (5) \\
\hline Antibiotic guideline for pneumonia ${ }^{33}$ & Mortality & RR 0.89 (0.82 to 0.97$)$ & 22526 & 4 & RCT (1); CBA (3) \\
\hline Rapid response team ${ }^{71}$ & Mortality & RR 0.91 (0.85 to 0.97 ) & 209639 & 4 & $\begin{array}{l}\text { RCT (2); CBA (1); } \\
\text { ITS (1) }\end{array}$ \\
\hline Interdisciplinary team interventions ${ }^{76}$ & Mortality & wRR 0.67 (0.45 to 0.99$)$ & 2640 & 2 & Non-RCT (2) \\
\hline Multicomponent interventions ${ }^{61}$ & Falls & $\operatorname{RaR} 0.69$ (0.49 to 0.96$)$ & 6478 & 4 & RCT (4) \\
\hline $\begin{array}{l}\text { Intervention effect estimates based on } \\
\text { meta-analysis with eligible and } \\
\text { non-eligible studies }\end{array}$ & Patient outcome & Effect size $(95 \% \mathrm{Cl})$ & $\begin{array}{l}\text { Sample size ( } \mathrm{n} \text { eligible } \\
\text { patients) and proportion of } \\
\text { eligible patients of all } \\
\text { patients }(\%)\end{array}$ & $\begin{array}{l}\text { Study size }(n) \text { and } \\
\text { proportion of eligible } \\
\text { studies }(n ; \%)\end{array}$ & $\begin{array}{l}\text { Designs of eligible } \\
\text { studies }(n)\end{array}$ \\
\hline Catheter reminder and stop order ${ }^{40}$ & Infections (CAUTI) & RR 0.72 (0.52 to 0.99$)$ & U & $8(2 ; 25)$ & $\begin{array}{l}\text { RCT (1); non-RCT } \\
\text { (1) }\end{array}$ \\
\hline Pharmacist interventions ${ }^{36}$ & Adverse drug events & OR 0.23 (0.11 to 0.48$)$ & 2794 (30.4) & $3(2 ; 66.7)$ & CBA (2) \\
\hline Care bundle and checklist ${ }^{39}$ & Infections (CLABSI) & OR 0.39 (0.33 to 0.46$)$ & $70358(2.8)$ & $41(5 ; 12.2)$ & BA (36); ITS (5) \\
\hline $\begin{array}{l}\text { Multicomponent interventions, including } \\
\text { early mobility, cognition and orientation }\end{array}$ & Delirium & OR 0.47 (0.38 to 0.58$)$ & $2914(68.3)$ & $11(7 ; 63.6)$ & $\begin{array}{l}\text { RCT (3); non-RCT } \\
\text { (4) }\end{array}$ \\
\hline Sepsis bundle ${ }^{41}$ & Mortality & OR 0.66 (0.61 to 0.72 ) & $11720(2.7)$ & $48(3 ; 6.3)$ & ITS (3) \\
\hline
\end{tabular}

${ }^{*} 17$ systematic reviews reported about 14 types of interventions.

†Studies with a design in accordance with methodological criteria of the Cochrane EPOC review group.

CCT, controlled (clinical) trial; CAUTI, catheter-associated urinary tract infection; CBA, controlled before after; CLABSI, central-line-associated bloodstream infection; EPOC, Effective Practice

and Organisation of Care; ITS, interrupted time series; NR, not reported; RaR, rate ratio; RCT, randomised controlled trial; RR, risk/relative ratio; U, unclear; wRR, weighted risk ratio. 
Box 1 Evidence-based effective patient-safety interventions $(n=14)$

Antibiotic guideline for pneumonia to reduce mortality rates.

Catheter reminder and stop order to reduce the risk for developing catheter-associated urinary tract infection.

Care bundles and checklists to reduce rates of central-lineassociated blood stream infections.

Clinical pathways to avoid complications.

Exercises to reduce the risk of falling.

Increasing the proportion of support staff to reduce mortality rates.

Interdisciplinary team interventions to reduce mortality rates.

Multicomponent interventions to reduce the risk of falling.

Multicomponent interventions to prevent delirium.

Nurse-led early-discharge planning programmes to reduce mortal-

ity rates.

Pharmacist interventions to prevent adverse drug events.

Rapid response team to reduce the risk for cardiopulmonary arrest and reduce mortality rates.

Sepsis bundle to reduce mortality rates.

Surgical safety checklist to reduce the risk for surgical-site infections and reduce mortality rates.

patient-safety improvement is still not strong. Furthermore, our findings are in contrast to the findings of previous research on this topic. Shekelle et $a l^{33}$ strongly supported the adoption of 10 patient-safety practices, including hand-hygiene strategies, the do-not-use list for hazardous abbreviations and multicomponent interventions to reduce pressure ulcers. We found limited support for the effectiveness of these interventions while finding strong support for delirium prevention interventions and rapid response teams. Our review placed more emphasis on assessing interventions on the basis of patient outcomes (ie, reduced adverse event and mortality rates) and testing within high-quality designs; this emphasis on the quality of studies produces a very different assessment of which safety interventions are most beneficial for patients and which should be implemented.

Evidence is still lacking for medication reconciliation and several interventions to improve the safety of clinical handover or discharge of hospitalised patients, which are incorporated in national and international patientsafety campaigns and are recommended by the WHO. ${ }^{84}$ However, the results of our review showed that by looking strictly at patient outcomes and only including high-quality studies, the evidence that these interventions reduce adverse event or mortality rates remains incomplete.

The lack of evidence for patient-safety interventions does not mean that these interventions do not work; it primarily addresses the lack of valid effect. Policymakers and clinicians show good intentions by implementing ambitious patient-safety programmes and investments of resources. However, implementing unproven interventions can lead to the opposite of what is intended with patient-safety improvements: waste of resources, energy and enthusiasm. ${ }^{85}{ }^{86}$ In times of limited resources, we concur with Shekelle et al and underscore previous, urgent calls for more research on the effectiveness of patient-safety interventions. ${ }^{7} 1283858788$ Patient-safety interventions should be tested on their effectiveness based on the same high-quality standards used for drug studies. $^{3} 89$

This systematic review has several limitations. First, we did not retrieve data from the primary studies; instead, we used the information reported by the authors on aspects, such as the description of the interventions and reported outcomes. As a result, the information for some patient-safety interventions and outcomes reported in our systematic review is limited. However, by focusing on the results of the systematic reviews rather than each individual primary study, we were able to obtain a broad overview of the field of patient safety. ${ }^{90}$ Second, the found estimates of effectiveness of patient-safety interventions might vary across contexts, such as small versus large hospitals, academically affiliated hospitals versus those that are not and the availability of factors that stimulate successful implementation of interventions, for example, strong leadership and an electronic patient record. ${ }^{91}$ Third, in two-thirds of the included systematic reviews, publication bias was not assessed (see online supplementary appendix 7), meaning that the pooled rates in these reviews may present an overestimation of the effect size. ${ }^{92}$ Fourth, in this study, valuable narrative syntheses from systematic reviews may have been under-reported, because we focused on the quantitative evidence of safety interventions. The large amount of eligible systematic reviews and subsequent data from primary studies restricted us to focus on the results from meta-analyses, which are widely considered as the highest level of evidence for the effectiveness of interventions (Oxford Centre for Evidence-Based MedicineLevels of Evidence). Fifth, the focus of our systematic review was to summarise quantitative evidence for existing patient-safety interventions. A limitation of this approach is that the found statistically significant effect measures may not be clinically significant and, vice versa, effects that are clinically relevant may not be statistically significant and were not captured in our systematic review.

In conclusion, patient-safety interventions are implemented worldwide, even though evidence for these interventions remains incomplete. A major cause for this problem is the lack of high-quality studies in which interventions are evaluated on their effects. To contribute to evidence-based patient safety, interventions need to be evaluated based on high-quality research standards, including experimental research designs, measured outcomes at the patient level and description of the intervention, implementation process and context in detail. Description of these aspects is necessary to know which factors lead to optimal effects and how to replicate the patient-safety intervention in practice. ${ }^{93} 94$ 
Policymakers and clinicians should stop taking shortcuts but need to spend more time and money conducting high-quality research on the effectiveness of patient-safety interventions to establish progress in patient safety.

Contributors MZ, GH, CV and HW contributed to the design of the study. MZ, GH and WG did the literature search, reviewed the studies for inclusion, assessed the included studies, extracted and analysed the data. MZ, GH and WG drafted the manuscript. CV and HW revised the manuscript critically for important intellectual content. All authors read and approved the final manuscript. MZ is the guarantor. The guarantor affirms that the manuscript is an honest, accurate and transparent account of the study being reported; that no important aspects of the study have been omitted; and that any discrepancies from the study as planned (and, if eligible, registered) have been explained.

Funding MZ has been supported by a research fellowship sponsored by ZonMw, The Netherlands Organisation for Health Research and Development (grant number 170996006). The funding source did not participate in the study's conception, data collection, analyses, manuscript preparation, decision to submit the manuscript for publication or any other part of the study.

Competing interests None declared.

Provenance and peer review Not commissioned; externally peer reviewed.

Data sharing statement No additional data are available.

Open Access This is an Open Access article distributed in accordance with the Creative Commons Attribution Non Commercial (CC BY-NC 4.0) license, which permits others to distribute, remix, adapt, build upon this work noncommercially, and license their derivative works on different terms, provided the original work is properly cited and the use is non-commercial. See: http:// creativecommons.org/licenses/by-nc/4.0/

\section{REFERENCES}

1. de Vries EN, Ramrattan MA, Smorenburg SM, et al. The incidence and nature of in-hospital adverse events: a systematic review. Qual Saf Health Care 2008;17:216-23.

2. Landrigan CP, Parry GJ, Bones CB, et al. Temporal trends in rates of patient harm resulting from medical care. $N$ Engl J Med 2010;363:2124-34.

3. Shojania KG. Conventional evaluations of improvement interventions: more trials or just more tribulations? BMJ Qual Saf 2013;22:881-4

4. Wang $\mathrm{Y}$, Eldridge $\mathrm{N}$, Metersky ML, et al. National trends in patient safety for four common conditions, 2005-2011. N Engl J Med 2014;370:341-51.

5. Duckers M, Faber M, Cruijsberg J, et al. Safety and risk management interventions in hospitals: a systematic review of the literature. Med Care Res Rev 2009;66(6 Suppl):90S-119S.

6. Etchells E, Koo M, Daneman N, et al. Comparative economic analyses of patient safety improvement strategies in acute care: a systematic review. BMJ Qual Saf 2012;21:448-56.

7. Wachter RM, Pronovost $P$, Shekelle $P$. Strategies to improve patient safety: the evidence base matures. Ann Intern Med 2013;158 (Pt 1):350-2.

8. Dy SM, Taylor SL, Carr LH, et al. A framework for classifying patient safety practices: results from an expert consensus process. BMJ Qual Saf 2011;20:618-24.

9. Ovretveit J. Leading evidence informed value improvement in health care. Chichester, UK: Kingsham Press, 2009

10. Shekelle PG, Pronovost P, Wachter RM, et al. Assessing the evidence for context-sensitive effectiveness and safety of patient safety practices: developing criteria. Rockville: Agency for Healthcare Research and Quality, 2010.

11. Shojania KG, Duncan BW, McDonald KM, et al. Safe but sound: patient safety meets evidence-based medicine. JAMA 2002;288:508-13.

12. WHO. The research priority setting working group. Summary of the evidence on patient safety: implications for research. Geneva: World Health Organization Alliance for Patient Safety, 2008.

13. Ovretveit J. Which interventions are effective for improving patient safety: a review of research evidence. Stockholm: Karolinska Institute, Medical Management Centre, 2005.
14. Shekelle PG, Wachter RM, Pronovost $\mathrm{P}$, et al. Making health care safer II: an updated critical analysis of the evidence for patient safety practices. Comparative effectiveness review No. 211. Rockville: Agency for Healthcare Research and Quality, 2013.

15. Wong J, Beglaryan $\mathrm{H}$. Strategies for hospitals to improve patient safety: a review of the research. The Change Foundation, 2004.

16. Smith V, Devane D, Begley CM, et al. Methodology in conducting systematic review of systematic reviews of healthcare interventions. BMC Med Res Methodol 2011;11:15.

17. Liberati A, Altman DG, Tetzlaff J, et al. The PRISMA statement for reporting systematic reviews and meta-analyses of studies that evaluate health care interventions: explanation and elaboration. $J$ Clin Epidemiol 2009;62:e1-34

18. Shea BJ, Hamel C, Wells GA, et al. AMSTAR is a reliable and valid measurement tool to assess the methodological quality of systematic reviews. J Clin Epidemiol 2009;62:1013-20.

19. Tanon AA, Champagne F, Contandriopoulos AP, et al. Patient safety and systematic reviews: finding papers indexed in MEDLINE, EMBASE and CINAHL. Qual Saf Health Care 2010;19: 452-61.

20. Cochrane Handbook. What study designs should be included in an EPOC review and what should they be called? 2016. http://epoc. cochrane.org/epoc-specific-resources-review-authors

21. Sharif $\mathrm{MO}$, Janjua-Sharif $\mathrm{FN}$, Ali $\mathrm{H}$, et al. Systematic reviews explained: AMSTAR - how to tell the good from the bad and the ugly. Oral Health Dent Manag 2013;12:9-16.

22. Wolfstadt JI, Gurwitz JH, Field TS, et al. The effect of computerized physician order entry with clinical decision support on the rates of adverse drug events: a systematic review. J Gen Intern Med 2008;23:451-8.

23. Maaskant JM, Vermeulen $\mathrm{H}$, Apampa B, et al. Interventions for reducing medication errors in children in hospital. Cochrane Database Syst Rev 2015;CD006208.

24. Holland R, Desborough J, Goodyer L, et al. Does pharmacist-led medication review help to reduce hospital admissions and deaths in older people? A systematic review and meta-analysis. $\mathrm{Br} \mathrm{J}$ Clin Pharmacol 2008;65:303-16.

25. Mueller SK, Sponsler KC, Kripalani S, et al. Hospital-based medication reconciliation practices: a systematic review. Arch Intern Med 2012;172:1057-69.

26. Christensen M, Lundh A. Medication review in hospitalised patients to reduce morbidity and mortality. Cochrane Database Syst Rev 2013;CD008986.

27. Hohl CM, Wickham ME, Sobolev B, et al. The effect of early in-hospital medication review on health outcomes: a systematic review. Br J Clin Pharmacol 2015;80:51-61.

28. Durieux P, Trinquart L, Colombet I, et al. Computerized advice on drug dosage to improve prescribing practice. Cochrane Database Syst Rev 2008;CD002894.

29. Gillaizeau F, Chan E, Trinquart L, et al. Computerized advice on drug dosage to improve prescribing practice. Cochrane Database Syst Rev 2013;CD002894.

30. Bayoumi I, Al Balas M, Handler SM, et al. The effectiveness of computerized drug-lab alerts: a systematic review and meta-analysis. Int J Med Inform 2014;83:406-15.

31. Kaboli PJ, Hoth AB, McClimon BJ, et al. Clinical pharmacists and inpatient medical care: a systematic review. Arch Intern Med 2006;166:955-64.

32. Manias $\mathrm{E}$, Williams $\mathrm{A}$, Liew $\mathrm{D}$. Interventions to reduce medication errors in adult intensive care: a systematic review. $\mathrm{Br} \mathrm{J}$ Clin Pharmacol 2012;74:411-23.

33. Davey P, Brown E, Charani E, et al. Interventions to improve antibiotic prescribing practices for hospital inpatients. Cochrane Database Syst Rev 2013;CD003543.

34. Patterson SM, Cadogan CA, Kerse N, et al. Interventions to improve the appropriate use of polypharmacy for older people. Cochrane Database Syst Rev 2014;CD008165.

35. Ensing HT, Stuijt CC, van den Bemt BJ, et al. Identifying the optimal role for pharmacists in care transitions: a systematic review. J Manag Care Spec Pharm 2015;21:614-36.

36. Wang T, Benedict N, Olsen KM, et al. Effect of critical care pharmacist's intervention on medication errors: a systematic review and meta-analysis of observational studies. J Crit Care 2015;30:1101-6.

37. Flodgren G, Conterno LO, Mayhew A, et al. Interventions to improve professional adherence to guidelines for prevention of device-related infections. Cochrane Database Syst Rev 2013; CD006559

38. Jansson $M$, Kääriäinen $M$, Kyngas $H$. Effectiveness of educational programmes in preventing ventilator-associated pneumonia: a systematic review. J Hosp Infect 2013;84:206-14. 
39. Blot K, Bergs J, Vogelaers D, et al. Prevention of central line-associated bloodstream infections through quality improvement interventions: a systematic review and meta-analysis. Clin Infect Dis 2014;59:96-105.

40. Meddings J, Rogers MA, Krein SL, et al. Reducing unnecessary urinary catheter use and other strategies to prevent catheter-associated urinary tract infection: an integrative review. BMJ Qual Saf 2014;23:277-89.

41. Damiani $E$, Donati $A$, Serafini $G$, et al. Effect of performance improvement programs on compliance with sepsis bundles and mortality: a systematic review and meta-analysis of observational studies. PLOS ONE 2015;10:e0125827.

42. Silvestri L, Petros AJ, Sarginson RE, et al. Handwashing in the intensive care unit: a big measure with modest effects. $J$ Hosp Infect 2005;59:172-9.

43. Gould DJ, Moralejo D, Drey N, et al. Interventions to improve hand hygiene compliance in patient care. Cochrane Database Syst Rev 2010;CD005186.

44. Safdar N, Abad C. Educational interventions for prevention of healthcare-associated infection: a systematic review. Crit Care Med 2008;36:933-40.

45. Cole MG, Primeau FJ, Elie LM. Delirium: prevention, treatment, and outcome studies. J Geriatr Psychiatry Neurol 1998;11:126-37; discussion $57-8$

46. Milisen $\mathrm{K}$, Lemiengre $\mathrm{J}$, Braes $\mathrm{T}$, et al. Multicomponent intervention strategies for managing delirium in hospitalized older people: systematic review. J Adv Nurs 2005;52:79-90.

47. Hempenius L, van Leeuwen BL, van Asselt DZ, et al. Structured analyses of interventions to prevent delirium. Int J Geriatr Psychiatry 2011;26:441-50.

48. Reston JT, Schoelles KM. In-facility delirium prevention programs as a patient safety strategy: a systematic review. Ann Intern Med 2013;158(Pt 2):375-80.

49. Collinsworth AW, Priest EL, Campbell CR, et al. A review of multifaceted care approaches for the prevention and mitigation of delirium in intensive care units. $J$ Intensive Care Med 2016;31:127-41.

50. Hshieh TT, Yue J, Oh E, et al. Effectiveness of multicomponent nonpharmacological delirium interventions: a meta-analysis. JAMA Intern Med 2015;175:512-20.

51. Martinez F, Tobar C, Hill N. Preventing delirium: should non-pharmacological, multicomponent interventions be used? A systematic review and meta-analysis of the literature. Age Ageing 2015;44:196-204.

52. Griffiths $\mathrm{P}$, Edwards M, Forbes $\mathrm{A}$, et al. Post-acute intermediate care in nursing-led units: a systematic review of effectiveness. Int $J$ Nurs Stud 2005;42:107-16.

53. Conroy SP, Stevens T, Parker SG, et al. A systematic review of comprehensive geriatric assessment to improve outcomes for frail older people being rapidly discharged from acute hospital: 'interface geriatrics'. Age Ageing 2011;40:436-43.

54. Niven DJ, Bastos JF, Stelfox HT. Critical care transition programs and the risk of readmission or death after discharge from an ICU: a systematic review and meta-analysis. Crit Care Med 2014;42:179-87.

55. Rennke S, Nguyen OK, Shoeb MH, et al. Hospital-initiated transitional care interventions as a patient safety strategy: a systematic review. Ann Intern Med 2013;158(Pt 2):433-40.

56. Shepperd S, Lannin NA, Clemson LM, et al. Discharge planning from hospital to home. Cochrane Database Syst Rev 2013; CD000313.

57. Lowthian JA, McGinnes RA, Brand CA, et al. Discharging older patients from the emergency department effectively: a systematic review and meta-analysis. Age Ageing 2015;44:761-70.

58. Zhu QM, Liu J, Hu HY, et al. Effectiveness of nurse-led early discharge planning programmes for hospital inpatients with chronic disease or rehabilitation needs: a systematic review and meta-analysis. J Clin Nurs 2015;24:2993-3005.

59. Oliver D, Connelly JB, Victor CR, et al. Strategies to prevent falls and fractures in hospitals and care homes and effect of cognitive impairment: systematic review and meta-analyses. BMJ 2007;334:82.

60. Coussement J, De Paepe L, Schwendimann R, et al. Interventions for preventing falls in acute- and chronic-care hospitals: a systematic review and meta-analysis. J Am Geriatr Soc 2008;56:29-36.

61. Cameron ID, Gillespie LD, Robertson MC, et al. Interventions for preventing falls in older people in care facilities and hospitals. Cochrane Database Syst Rev 2012;12:CD005465.

62. Miake-Lye IM, Hempel S, Ganz DA, et al. Inpatient fall prevention programs as a patient safety strategy: a systematic review. Ann Intern Med 2013;158(Pt 2):390-6.
63. Chen AF, Wessel CB, Rao N. Staphylococcus aureus screening and decolonization in orthopaedic surgery and reduction of surgical site infections. Clin Orthop Relat Res 2013;471:2383-99.

64. Howell AM, Panesar SS, Burns EM, et al. Reducing the burden of surgical harm: a systematic review of the interventions used to reduce adverse events in surgery. Ann Surg 2014;259: 630-41.

65. Hempel S, Maggard-Gibbons M, Nguyen DK, et al. Wrong-site surgery, retained surgical items, and surgical fires : a systematic review of surgical never events. JAMA Surg 2015;150:796-805.

66. Bergs J, Hellings J, Cleemput I, et al. Systematic review and meta-analysis of the effect of the World Health Organization surgical safety checklist on postoperative complications. Br J Surg 2014;101:150-8.

67. Algie CM, Mahar RK, Wasiak J, et al. Interventions for reducing wrong-site surgery and invasive clinical procedures. Cochrane Database Syst Rev 2015;CD009404.

68. Esmonde L, McDonnell A, Ball C, et al. Investigating the effectiveness of critical care outreach services: a systematic review. Intensive Care Med 2006;32:1713-21.

69. Chan PS, Jain R, Nallmothu BK, et al. Rapid response teams: a systematic review and meta-analysis. Arch Intern Med 2010;170:18-26.

70. Massey D, Aitken LM, Chaboyer W. Literature review: do rapid response systems reduce the incidence of major adverse events in the deteriorating ward patient? $J$ Clin Nurs 2010;19:3260-73.

71. Maharaj R, Raffaele I, Wendon J. Rapid response systems: a systematic review and meta-analysis. Crit Care 2015;19:254.

72. Kahn SR, Morrison DR, Cohen JM, et al. Interventions for implementation of thromboprophylaxis in hospitalized medical and surgical patients at risk for venous thromboembolism. Cochrane Database Syst Rev 2013:CD008201.

73. Lau BD, Haut ER. Practices to prevent venous thromboembolism: a brief review. BMJ Qual Saf 2014;23:187-95.

74. Reed DA, Fletcher KE, Arora VM. Systematic review: association of shift length, protected sleep time, and night float with patient care, residents' health, and education. Ann Intern Med 2010;153:829-42

75. Butler M, Collins R, Drennan J, et al. Hospital nurse staffing models and patient and staff-related outcomes. Cochrane Database Syst Rev 2011;CD007019.

76. Pannick S, Davis R, Ashrafian $\mathrm{H}$, et al. Effects of interdisciplinary team care interventions on general medical wards: a systematic review. JAMA Intern Med 2015;175:1288-98.

77. Sullivan N, Schoelles KM. Preventing in-facility pressure ulcers as a patient safety strategy: a systematic review. Ann Intern Med 2013;158(Pt 2):410-16.

78. Naylor CJ, Griffiths RD, Fernandez RS. Does a multidisciplinary total parenteral nutrition team improve patient outcomes? A systematic review. JPEN J Parenter Enteral Nutr 2004;28:251-8.

79. Rotter T, Kinsman L, James E, et al. Clinical pathways: effects on professional practice, patient outcomes, length of stay and hospital costs. Cochrane Database Syst Rev 2010;CD006632.

80. Weaver SJ, Lubomksi LH, Wilson RF, et al. Promoting a culture of safety as a patient safety strategy: a systematic review. Ann Intern Med 2013;158(Pt 2):369-74.

81. Flodgren G, Pomey MP, Taber SA, et al. Effectiveness of external inspection of compliance with standards in improving healthcare organisation behaviour, healthcare professional behaviour or patient outcomes. Cochrane Database Syst Rev 2011; CD008992.

82. Whitlock EP, Lin JS, Chou R, et al. Using existing systematic reviews in complex systematic reviews. Ann Intern Med 2008:148:776-82

83. Shekelle PG, Pronovost PJ, Wachter RM, et al. The top patient safety strategies that can be encouraged for adoption now. Ann Intern Med 2013;158(Pt 2):365-8.

84. WHO. Patient safety. Secondary Patient safety, 2015. http://www. who.int/patientsafety/en/

85. Marshall M, Pronovost P, Dixon-Woods M. Promotion of improvement as a science. Lancet 2013;381:419-21.

86. Rennenberg RJ. [Increasing the safety of healthcare: valuable, but at what cost?]. Ned Tijdschr Geneeskd 2013;157:A6044.

87. Shojania KG, Thomas EJ. Trends in adverse events over time: why are we not improving? BMJ Qual Saf 2013:22:273-7.

88. Wachter RM. Patient safety at ten: unmistakable progress, troubling gaps. Health Aff (Millwood) 2010;29:165-73.

89. Grol R, Baker R, Moss F. Quality improvement research: understanding the science of change in health care. London: BMJ Books, 2004. 
90. Cheung A, Weir M, Mayhew A, et al. Overview of systematic reviews of the effectiveness of reminders in improving healthcare professional behavior. Syst Rev 2012;1:36.

91. Grol R, Grimshaw J. From best evidence to best practice: effective implementation of change in patients' care. Lancet 2003;362:1225-30.

92. Higgins JPT, Green S. Cochrane handbook for systematic reviews of interventions. Secondary Cochrane handbook for systematic reviews of interventions, 2011. http://handbook.cochrane.org/front_ page.htm

93. Hulscher ME, Laurant MG, Grol RP. Process evaluation on quality improvement interventions. Qual Saf Health Care 2003;12:40-6.

94. Shekelle PG, Pronovost PJ, Wachter RM, et al. Advancing the science of patient safety. Ann Intern Med 2011;154:693-6. 